\title{
Looking for the Right Swipe: Gender Differences in Self-Presentation on Tinder Profiles
}

\author{
Gordon P. D. INGRAM ${ }^{\mathrm{a}, 1}$, M. Isabela ENCISO ${ }^{\mathrm{a}}$, Nathalia ERASO ${ }^{\mathrm{a}}$, \\ Maria Jose GARCIA ${ }^{\mathrm{a}}$ and Antonio OLIVERA-LA ROSA ${ }^{\mathrm{b}}$ \\ ${ }^{a}$ Universidad de los Andes, Colombia \\ ${ }^{\mathrm{b}}$ Universidad Católica Luis Amigó, Medellín, Colombia
}

\begin{abstract}
Tinder has become a popular online dating tool for people looking for either short- or long-term relationships. In this study we build on existing research on gender differences in the motivations of Tinder users, by analyzing gender differences in self-presentation. We predicted that women would try to attract men to right-swipe (i.e., potentially match) their profiles through largely visual means; while men would put more emphasis on showing off their skills and interests. In a sample of 300 randomly obtained Tinder profiles ( 150 heterosexual females and 150 heterosexual males), half from Colombia and half from the USA, we found mixed support for our hypotheses. There was no significant difference between numbers of photos uploaded by women and men, but certain types of photos were unique to one gender. Links to other social networks did not differ much between genders; however, men tended to include more textual information in their profiles.
\end{abstract}

Keywords. Gender differences, Instagram, online dating, self-presentation, selfies, Spotify, Tinder

\footnotetext{
${ }^{1}$ Corresponding Author, Gordon P. D. Ingram, Department of Psychology, Universidad de los Andes, Carrera
} 1 \# 18A-12, Bogotá, Colombia; E-mail: gp.ingram@uniandes.edu.co. 


\section{Introduction and Hypotheses}

Although stereotyped as the preserve of people looking for casual sex, Tinder has become a popular online dating tool for people looking for either short- or long-term relationships [1,2,3], with an estimated 50 million users in more than 190 countries, and 10 million daily active users. In a uses and gratifications study, [1] showed that Casual Sex was just one of several classes of motivations for using Tinder, while Love was more common; and while $46 \%$ of users had gone on a date with a Tinder match, only $19 \%$ had taken part in a one-night stand with a match. It is thus reasonable to suppose that patterns of gender differences found in other online dating apps and sites (reviewed in [4]) will also apply to Tinder. In particular, theory predicts differences in the gender mix of people who use the site for short-term and long-term mating purposes, as well as differences in the strategies that different genders use to attract a mate.

Indeed, gender differences in Tinder use are very apparent: [5] found that men tended to use Tinder for sex, travel and starting a relationship, whereas women used it more for friendship and self-validation. Similarly, [1] found that men were more likely than women to use Tinder for casual sex. This is in line with evolutionary psychological studies of online dating [4] and of gender differences in social network use [6]. In addition to cultural factors, a successful evolutionary explanation of sex differences in self-presentation generally is the sexual strategies theory of Buss and Schmitt [7]. This theory posits that due to their higher obligatory investment in offspring, women have less to gain from a short-term mating strategy and should therefore be more conservative and less risky in their mating choices. [8] reviewed evidence suggesting that men were more likely to initiate "sexting" (exchanges of sexually explicit text messages) than women were. While this finding might be expected to generalize to the initiation of chats on Tinder, this was not the hypothesis examined in the current study. Instead, we sought to examine gender differences in self-presentation on Tinder profiles.

While separate from the question of who initiates sexual interactions, gender differences in self-presentation are also related to issues of parental investment and sexual strategies. There is a kind of complementarity between self-presentation and attraction, in that women tend to try to attract men primarily through visual means, emphasizing their value as short-term mating prospects, whereas men often try to convince women that they are reliable, low-risk, long-term mating prospects, a proposition that may involve a certain amount of verbal persuasion. Although Tinder is primarily a visual application, both visual and verbal means of self-presentation are available to users. Interaction between users starts with one user viewing a photo of another who lives nearby, along with some very basic biographical information (their age and the university where they were educated). If the user likes the look of the photo, he/she swipes right; if not, he/she swipes left [3]. If at some other time the user in the photo sees the photo of the first user in their search results and swipes right on someone who has previously swiped right on them, a "match" occurs, and conversations between the two often begin. However, in certain cases a user is not sure from the first photo whether they will make a right or left swipe. They can therefore dig deeper into the other user's profile and unearth more information if it is provided, including additional photos, some resumé-type biographical information, free text comments (known as the "profile description"), and sometimes links to other social media accounts, notably Instagram (for even more photos) and Spotify (for the user's favorite music).

We therefore hypothesized that women would try to attract men to right-swipe (i.e., potentially match) their profiles through largely visual means; while men would try to 
do so more by showing off their skills and interests in the textual information fields and by linking to Spotify accounts. In particular, showing pictures of the whole body should be more important for females who want to demonstrate their potential suitability as short-term mates, according to a large body of work on the importance of the waist/hip ratio in female attractiveness [9]. We predicted that $(\mathrm{H} 1)$ females would upload more photos to their Tinder profile than males; (H2) a higher proportion of photos would be of the whole body in females; (H3) females would more frequently link to their Instagram account than males, in order to provide even more photographic material for male consumers; and (H4) males would more often link to their Spotify account than females, in order to demonstrate an important non-visual aspect of their personal identity

\section{Method}

These predictions were tested by creating one male profile and one female on Tinder, and then selecting and classifying the first 300 Tinder profiles (150 heterosexual females and 150 heterosexual males) that appeared. Half of these profiles were collected from Colombia and half from the USA, with equal numbers of females and males in each country. We coded each profile for number of photos included and whether Instagram and/or Spotify accounts were linked. We also developed a scheme for categorizing the photos that had been uploaded, assigning each of them a value of Yes/No for each of the following variables: selfie; mirror selfie; travel (e.g. vacation shot); with friends; showing whole body; sports-related; and with pets.

\section{Results}

- $\mathrm{H} 1$ : females submitted a mean of 3.9 photos $(\mathrm{SD}=2.0)$, while males submitted $4.3(\mathrm{SD}=2.0)$, a non-significant difference $(t=1.2, p=.25)$

- $\quad H 2$ : pictures of the whole body and mirror selfies were unique to female profiles (see Table 1 below)

- H3: $39 \%$ of females and 31\% of males linked to their Instagram account, a nonsignificant difference $\left(\chi^{2}=2.1, p=.15\right)$

- H4: $35 \%$ of males but only $22 \%$ of females linked to their Spotify account, a significant difference $\left(\chi^{2}=6.5, p=.01\right)$

Table 1. Differing proportions of photos in each category (not exhaustive), split by gender $\left({ }^{* * *}\right.$ significant at the 0.001 level; ${ }^{* *}$ significant at the 0.01 level$)$.

\begin{tabular}{rcc}
\hline Photo category & Females $($ col \%) & Males (col \%) \\
\hline Selfies & 34 & 30 \\
Mirror selfies & $11^{* * *}$ & 0 \\
Travel & 5 & $15^{* *}$ \\
With friends & 5 & 5 \\
Body & $14^{* * *}$ & 0 \\
Sports & 0 & $6^{* *}$ \\
Pets & 0 & $6^{* *}$ \\
\hline
\end{tabular}

Notable unpredicted results included that pictures of sporting activities and pets were 
found only for males, although proportions were low $(6 \%)$ in both cases, so this difference may not be very reliable. Pictures taken while traveling (e.g., on vacation) were also significantly more common in men $\left(\chi^{2}=8.1, p=.004\right)$. We further found that men were much more likely than women to include a verbal profile description, and to include information about their college major. Results were broadly similar in both countries: given the relatively small samples $(n=75)$ when split by country and gender, we do not report country-specific analyses here.

\section{Discussion}

H1 was not supported: we found no significant difference between numbers of photos uploaded by women and men, with the trend even being in the opposite direction to the prediction. This is in contrast to studies of other social media such as Facebook in which female users typically upload many more photos than male users [4]. This discrepancy may be because Tinder initially asks users to upload a maximum of only six photos when they register with the app. While it is possible to upload more, the user interface for this is not intuitive and only $6 \%$ of our sample had done so. Future work could investigate whether the difference exists in dating apps/sites that make it easier for users to upload photos. In contrast, H2 was supported: men never uploaded mirror selfies or even other photos that showed their whole body. This suggests that rather than simple visual information such as photos in general marking a gender difference in sexual strategies, it may be certain types of visual information — such as waist-hip ratio [5] - that are important. Further research could focus on whether poses and clothing that heterosexual women use in photos on Tinder and other dating sites, compared with social networks where they are not specifically trying to attract men, serve to accentuate this ratio.

H3 was not supported, especially considering the slight female bias in Instagram's user base. Although H4 was superficially supported, correcting for the 55-45\% malefemale split in Spotify's user base would likely mean it was not supported either. In retrospect, we would expect much less of a gender difference in linking to other social network accounts than in text-based profile elements such as description of university major that allow males to demonstrate their earnings potential, or photographic elements that allow the same (such as travel pics) or demonstrate a more caring side (pictures with pets). However, these hypotheses did not occur to us before we carried out our exploratory analysis of Tinder profile elements. Since the results in these areas were not predicted, they need careful replication before firm conclusions can be drawn from them.

\section{References}

[1] S. R. Sumter, L Vandenbosch \& L. Ligtenberg, Love me Tinder: untangling emerging adults' motivations for using the dating application Tinder, Telematics and Informatics 34 (2017), 67-78.

[2] L. E. LeFebvre, Swiping me off my feet: explicating relationship initiation on Tinder, Journal of Social and Personal Relationships 35 (2018), 1205-1229.

[3] G. Orosz et al., The personality, motivational, and need-based background of problematic Tinder use, Journal of Behavioral Addictions 7 (2018), 301-316.

[4] O. Abramova, A. Baumann, H. Krasnova \& P. Buxmann, Gender differences in online dating: what do we know so far? a systematic literature review, HICSS 49 (2016), DOI: 10.1109/HICSS.2016.481.

[5] G. Ranzini \& C. Lutz,. Love at first swipe? Explaining Tinder self-presentation and motives, Mobile Media and Communication 5 (2017), 80-101. 
[6] F. T. McAndrew \& H. S. Jeong, Who does what on Facebook? age, sex, and relationship status as predictors of Facebook use, Computers in Human Behavior 28 (2012), 2359-2365.

[7] D. M. Buss \& D. P. Schmitt, Sexual strategies theory: an evolutionary perspective on human mating, Psychological Review 100 (1993), 204-232.

[8] J. R. Piazza \& G. P. D. Ingram, Evolutionary cyberpsychology 2.0, in Evolutionary Perspectives on Social Psychology, pp. 159-174, Springer, Cham, Switzerland, 2015.

[9] D. Singh, B. J. Dixson, T. S. Jessop, B. Morgan \& A. F. Dixson, Cross-cultural consensus for waist-hip ratio and women's attractiveness, Evolution and Human Behavior 31 (2010), 176-181. 